\title{
A CHARACTERIZATION OF RATIONAL NUMBERS BY p-ADIC RUBAN CONTINUED FRACTIONS
}

\author{
VICHIAN LAOHAKOSOL \\ (Received 24 October 1983; revised 11 May 1984) \\ Communicated by P. E. Blanksby
}

\begin{abstract}
A type of $p$-adic continued fraction first considered by A. Ruban is described, and is used to give a characterization of rational numbers.

1980 Mathematics subject classification (Amer. Math. Soc.): 10 F 45, 10 A 32, 10 F 20.
\end{abstract}

\section{Introduction}

Analogues for $p$-adic numbers of the classical continued fraction for real numbers have been studied by various authors, particularly Mahler. The $p$-adic algorithm which most closely mimics the classical algorithm via integer parts was mentioned in one of the earliest papers on the subject (Mahler (1934)) but was not pursued there because this algorithm does not yield very good approximations. The continued fraction based on this algorithm, here called the Ruban continued fraction, was developed by Ruban (1970), who showed $(1970,1973)$ on the other hand, that this continued fraction does have nice ergodic properties, and who also $(1972,1976)$ extended these results to a multi-dimensional $p$-adic analogue of Perron's algorithm. The Ruban continued fraction and the different but similar continued fraction developed by Schneider (1970) share the difficulty that there are rational numbers (including all negative rational numbers) possessing infinite continued fractions, and Schneider's paper raised the problem of characterising the rational numbers in terms of their $p$-adic continued fractions. The first part of

(C) 1985 Australian Mathematical Society $0263-6115 / 85 \$ A 2.00+0.00$ 
my Master's thesis (1978), written under the supervision of Dr. Jane Pitman, consisted of a survey and comparison of the various $p$-adic continued fractions and included, in particular, some work on the problem of characterising the rationals, which, however, was not resolved there.

Recently, I have discovered that Bundschuh (1977) had solved the problem of characterising the rationals in terms of their Schneider $p$-adic continued fraction. In the present note, I shall obtain a similar characterisation of the rationals in terms of their Ruban p-adic continued fraction using ideas based on those of Bundschuh. This main result is given in Section 3, and is preceded by an introduction to the Ruban continued fraction in Section 2.

In what follows, $p$ denotes a fixed rational prime, $|\cdot|_{p}$ its corresponding $p$-adic valuation so normalized $|p|_{p}=1 / p, \mathbf{Z}_{p}$ the ring of $p$-adic integers, $p \mathbf{Z}_{p}=\{p z ; z$ $\left.\in \mathbf{Z}_{p}\right\}$, and $|\cdot|$ the usual absolute valuation.

The abbreviation RCF stands for $p$-adic Ruban continued fractions.

\section{2. p-adic Ruban continued fractions (RCF)}

With no loss of generality, as will be clear from the construction, we shall first describe the $p$-adic continued fraction of Ruban (1970) of an element in $p \mathbf{Z}_{p}$. Let $\xi \in p \mathbf{Z}_{p} \backslash\{0\}$. Since $\left|\xi^{-1}\right|_{p}>1$, then let the unique representation of $\xi^{-1}$ be

$$
\xi^{-1}=c_{-m} p^{-m}+c_{-m+1} p^{-m+1}+\cdots+c_{-1} p^{-1}+c_{0}+c_{1} p+\cdots,
$$

where $\left|\xi^{-1}\right|_{p}=p^{m}$ and $c_{i} \in\{0,1, \ldots, p-1\}$ for all $i \geqslant-m$, with $c_{-m} \neq 0$. Define

$$
\begin{gathered}
\left\langle\xi^{-1}\right\rangle=\text { fractional part of } \xi^{-1}:=c_{-m} p^{-m}+\cdots+c_{0}, \\
\left(\xi^{-1}\right)=\text { integral part of } \xi^{-1}:=c_{1} p+c_{2} p^{2}+\cdots .
\end{gathered}
$$

To such $\xi$, the integral and fractional parts of $\xi^{-1}$ are uniquely determined, and so we can uniquely write $\xi^{-1}=\left\langle\xi^{-1}\right\rangle+\left(\xi^{-1}\right)$. The algorithm proceeds as follows: write $\xi^{-1}=b_{0}+\xi_{1}$, where $b_{0}=\left\langle\xi^{-1}\right\rangle, \xi_{1}=\left(\xi^{-1}\right)$. If $\xi_{1}=0$, the algorithm stops. Otherwise, since $\left|\xi_{1}\right|_{p}<1$, by repeating the steps just described, we can uniquely write $\xi_{1}^{-1}=b_{1}+\xi_{2}$, where $b_{1}=\left\langle\xi_{1}^{-1}\right\rangle, \xi_{2}=\left(\xi_{1}^{-1}\right)$. Again, if $\xi_{2}=0$, we stop, otherwise we proceed in the same manner. Since the $b_{i}$ 's $(i \geqslant 0)$ so obtained are unique, each $\xi \in p \mathbf{Z}_{p} \backslash\{0\}$ has a unique RCF ( $p$-adic Ruban continued fraction) of the form

$$
\frac{1}{b_{0}+} \frac{1}{b_{1}+} \frac{1}{b_{2}+} \cdots,
$$

(*) $\left\{\begin{array}{l}\text { where the } b_{i} \text { 's are of the form } c_{-m} p^{-m}+c_{-m+1} p^{-m+1}+\cdots+c_{0} \\ \text { for some positive integer } m \text {, and } c_{i} \in\{0,1, \ldots, p-1\} \\ \text { for all } i \text {, with } c_{-m} \neq 0 .\end{array}\right.$ 
Next, we define two sequences of rational numbers $\left\{A_{n}\right\}$ and $\left\{B_{n}\right\}$ by

$$
\begin{array}{ll}
A_{-1}=1, \quad B_{-1}=0, & A_{0}=0, \quad B_{0}=1, \\
A_{n+1}=b_{n} A_{n}+A_{n-1}, & B_{n+1}=b_{n} B_{n}+B_{n-1} \quad(n \geqslant 0) .
\end{array}
$$

It is easily checked that

$$
\frac{A_{n}}{B_{n}}=\frac{1}{b_{0}+} \frac{1}{b_{1}+} \cdots \frac{1}{b_{n-1}} \quad(n \geqslant 1),
$$

and, as usual, we call $A_{n} / B_{n}$ the $n$th convergent of the RCF of $\xi$.

We must show that such an RCF does indeed converge to $\xi$. Let $n$ be a positive integer. Then at the $n$th step of construction we have

$$
\xi_{n-1}^{-1}=b_{n-1}+\xi_{n},
$$

where $\xi_{n-1}, \xi_{n} \in p \mathbf{Z}_{p}$, and $b_{n-1}$ is of the form stated earlier. If $\xi_{n}=0$ for some $n$, then the RCF is finite and is equal to $\xi$. If $\xi_{n} \neq 0$ for every positive integer $n$, then by induction

$$
\xi-\frac{A_{n}}{B_{n}}=\frac{(-1)^{n}}{B_{n}\left(\xi_{n}^{-1} B_{n}+B_{n-1}\right)} \quad(n \geqslant 1) .
$$

Since $\left|b_{i}\right|_{p}>1$, by induction we see that from (2.1) we have

$$
\left|B_{n}\right|_{p}=\left|b_{0} b_{1} \cdots b_{n-1}\right|_{p} \quad(n \geqslant 1) .
$$

Also, (2.2) implies $\left|\xi_{n}^{-1}\right|_{p}=\left|b_{n}\right|_{p}(n \geqslant 1)$. Consequently,

$$
\left|\xi-A_{n} / B_{n}\right|_{p}=\left|b_{0}^{2} b_{1}^{2} \cdots b_{n-1}^{2} b_{n}\right|_{p}^{-1} \rightarrow 0 \quad(n \rightarrow \infty),
$$

that is, the RCF converges to $\xi$.

Given an RCF of the form (2.0), by induction it easily follows that

$$
\frac{A_{n}}{B_{n}}-\frac{A_{n-1}}{B_{n-1}}=\frac{(-1)^{n-1}}{B_{n-1} B_{n}}
$$

and so

$$
\left|\frac{A_{n}}{B_{n}}-\frac{A_{n-1}}{B_{n-1}}\right|_{p}=\left|B_{n-1} B_{n}\right|_{p}^{-1}=\left|b_{0}^{2} b_{1}^{2} \cdots b_{n-2}^{2} b_{n-1}\right|_{p}^{-1} \rightarrow 0 \quad(n \rightarrow \infty),
$$

implying that the RCF converges to some $\xi \in p \mathbf{Z}_{p}$. This RCF is easily shown to be identical with the one obtained from $\xi$ by the Ruban algorithm. We summarize the result as follows.

THEOREM 1. To each $\xi \in p \mathbf{Z}_{p} \backslash\{0\}$, there corresponds a unique $R C F$ of the form (2.0) where the $b_{i}$ 's are of the form given in (*). This unique $R C F$ converges to $\xi$, so we write $\xi=\frac{1}{b_{0}+} \frac{1}{b_{1}+} \frac{1}{b_{2}+} \ldots$. Conversely, if $b_{i}$ 's are of the form $(*)$, then the continued fraction $\frac{1}{b_{0}+} \frac{1}{b_{1}+} \frac{1}{b_{2}+} \cdots$ is an RCF representing a unique number in $p \mathbf{Z}_{p} \backslash\{0\}$. 


\section{Characterization of rational numbers}

It is quite trivial that a finite $\mathrm{RCF}$ always represents a rational number. However, there exist infinitely many rational numbers with periodic RCF's, as the following example shows.

$$
\begin{gathered}
-p=\frac{1}{(p-1) p^{-1}+(p-1)+} \frac{1}{(p-1) p^{-1}+(p-1)+} \\
\frac{1}{(p-1) p^{-1}+(p-1)+\cdots,}
\end{gathered}
$$

and for any positive integer $s$, since

$$
-p^{-s}=(p-1) p^{-s}+(p-1) p^{-s+1}+\cdots+(p-1)+(-p),
$$

we have

$$
\begin{aligned}
& -p^{s}=\frac{1}{(p-1) p^{-s}+(p-1) p^{-s+1}+\cdots+(p-1)+} \\
& \frac{1}{(p-1) p^{-1}+(p-1)+} \frac{1}{(p-1) p^{-1}+(p-1)+\cdots,}
\end{aligned}
$$

where the RCF is periodic from the second fraction onwards with the above shape. Indeed, we prove that $\xi$ is rational if and only if its RCF either terminates or has a "tail" identical with the RCF of $-p$.

THEOREM 2. Let $\xi \in p \mathbf{Z}_{p} \backslash\{0\}$. Then $\xi$ is rational if and only if either the RCF of $\xi$ is finite, or the RCF of $\xi$ is periodic from a certain fraction onwards with the shape

$$
\frac{1}{(p-1) p^{-1}+(p-1)+} \frac{1}{(p-1) p^{-1}+(p-1)+} \cdots
$$

PRoof. If the RCF of $\xi$ is finite or periodic with such form, it is clear that $\xi$ is a rational number, so we need only prove the other implication.

Without any loss of generality, let $\xi$ be a rational number in $p \mathbb{Z}_{p} \backslash\{0\}$. Let also the RCF of $\xi$ be as in (2.0), where the $b_{i}$ 's are of the form (*).

At this point, we come across the fact that both the partial quotients $b_{i}$ 's and the numbers $A_{n}, B_{n}$ of the RCF of $\xi$ are rational, not integral, so we introduce an associated continued fraction

$$
\frac{\alpha_{0}}{\beta_{0}+} \frac{\alpha_{1}}{\beta_{1}+} \frac{\alpha_{2}}{\beta_{2}+} \cdots,
$$

where $\alpha_{0}=\left|b_{0}\right|_{p}, \alpha_{n}=\left|b_{n} b_{n-1}\right|_{p}(n \geqslant 1), \beta_{n}=b_{n}\left|b_{n}\right|_{p}(n \geqslant 0)$. 
Evidently, this associated continued fraction (3.1) has partial quotients $\alpha_{n}$ and $\beta_{n}$ being rational integers. It also follows easily that the RCF (2.0) and the associated continued fraction (3.1) are equivalent in the sense that both have the same $n$th convergents for every $n \geqslant 0$ (compare with Perron (1954), Chapter 1 ), so we may write

$$
\xi=\frac{1}{b_{0}+} \frac{1}{b_{1}+} \frac{1}{b_{2}+} \cdots=\frac{\alpha_{0}}{\beta_{0}+} \frac{\alpha_{1}}{\beta_{1}+} \frac{\alpha_{2}}{\beta_{2}+} \cdots .
$$

Now we unwind the associated continued fraction in the following manner.

Since $\xi$ is rational, we can write

$$
\xi=\frac{\alpha_{0}}{x_{0} / x_{1}}=\frac{\alpha_{0}}{\beta_{0}+\alpha_{1} \frac{x_{2}}{x_{1}}}=\frac{\alpha_{0}}{\beta_{0}+\frac{\alpha_{1}}{\beta_{1}+\alpha_{2} \frac{x_{3}}{x_{2}}}}=\cdots,
$$

where all $x_{n}$ are rational integers satisfying $\left(x_{n}, x_{n-1}\right)=\left(x_{n}, p\right)=\left(x_{n-1}, p\right)=1$. It is easily checked that for all $n, x_{n-1}=\beta_{n-1} x_{n}+\alpha_{n} x_{n+1}$, and it can also be shown by using the definitions of $\alpha_{n}$ and $\beta_{n}$ and the property (*) of $b_{n}$ that $1 \leqslant \beta_{n} \leqslant \alpha_{n+1}-1$. Thus for all $n, 0 \leqslant\left|x_{n+1}\right| \leqslant \max \left(\left|x_{n}\right|,\left|x_{n-1}\right|\right)$. Consequently, there is a fixed positive integer $N$ such that for all $n \geqslant N$, we have $\left|x_{n+1}\right|=\left|x_{n}\right|$. If there exists an integer $k>N$ such that $x_{k-1}=x_{k}$, then $-\alpha_{k} x_{k+1}=\left(\beta_{k-1}-1\right) x_{k}$, and so $\alpha_{k} \mid\left(\beta_{k-1}-1\right)$. But $\alpha_{k}>\alpha_{k}-2 \geqslant \beta_{k-1}-1$. Thus $\beta_{k-1}=1$, yielding $x_{k+1}=0$. Hence, the continued fraction is finite, and we have nothing to prove.

Therefore, we assume for all $n>N$ that $x_{n} \neq 0$ and $x_{n-1}=-x_{n}$. This yields $x_{n-1} / x_{n}=-1$. Consequently,

$$
\xi=\frac{\alpha_{0}}{\beta_{0}+} \frac{\alpha_{1}}{\beta_{1}+} \cdots \frac{\alpha_{N}}{\beta_{N}+\alpha_{N+1}(-1)}=\frac{1}{b_{0}+} \frac{1}{b_{1}+} \cdots \frac{1}{b_{N}+p^{s}(-1)},
$$

for some positive integer $s$. By appealing to the RCF of $-p^{s}$ in (3.0), the result is now evident.

We conclude this note with two remarks.

(i) The problem of deciding whether a quadratic ( $p$-adic) irrational number has a periodic $p$-adic continued fraction (either Schneider, or Ruban type) is still open, though the available work does not seem to support this view.

(ii) It would be of interest to extend the result of this paper to Ruban's multi-dimensional continued fraction.

\section{Acknowledgment}

I wish to thank the referee for comments and suggestions which greatly improved the presentation of this paper. 


\section{References}

P. Bundschuh (1977), ' $p$-adische kettenbrüche und irrationalität p-adischer zahlen', Elem. Math. 32, 36-40.

V. Laohakosol (1978), Two topics in p-adic approximation (M. Sc. thesis, University of Adelaide, South Australia).

K. Mahler (1934), 'Zur approximation p-adischer irrationalzahlen', Nieuw Arch. Wisk. 18, 22-34.

O. Perron (1954), Die lehre von den kettenbrüchen, Band II (Teubner, Stuttgart).

A. A. Ruban (1970), 'Some metric properties of p-adic numbers', Sibirsk. Mat. Ž. 11, 222-227. English translation: Siberian Math. J 11, 176-180.

A. A. Ruban (1972), 'The Perron algorithm for $p$-adic numbers and some of its ergodic properties', Dokl. Akad. Nauk SSSR 204, 45-48. English translation: Soviet Math. Dokl. 13, 606-609.

A. A. Ruban (1973), 'An invariant measure for a transformation related to Perron's algorithm for p-adic numbers', Dokl. Akad. Nauk SSSR 213, 536-537. English translation: Soviet Math. Dokl. 14, 1752-1754.

A. A. Ruban (1976), 'The entropy of a transformation connected with Perron's algorithm for $p$-adic numbers', Dokl. Akad. Nauk SSSR 227, 571-573. English translation: Soviet Math. Dokl. 17, 475-477.

Th. Schneider (1970), 'Über $p$-adische kettenbrüche', Symposia Mathematica 4, 181-189.

Department of Mathematics

Kasetsart University

Bangkok 10900

Thailand 